Article

\title{
Cluster Control for EVs Participating in Grid Frequency Regulation by Using Virtual Synchronous Machine with Optimized Parameters
}

\author{
Dongqi Liu \\ School of Electrical and Information Engineering, Changsha University of Science and Technology, \\ Changsha 410114, China; liu.dongqi@hotmail.com; Tel.: +86-185-0845-0006
}

Received: 17 March 2019; Accepted: 8 May 2019; Published: 10 May 2019

\begin{abstract}
In this paper, a control method for electric vehicles (EVs) participating in grid Frequency regulation is proposed. Firstly, considering dispatching large-scale electric vehicles, the K-means clustering algorithm is applied to cluster EVs with different battery state of charge and with different average vehicle daily travel miles. Then, for each class of electric vehicle group, a multi-objective optimization model considering reducing power imbalance and feeding the driving power demand for electric vehicles is proposed. Multi-Objective Particle Swarm Optimization (MOPSO) algorithm is applied to solve the optimization model and obtain the best control parameters for "virtual synchronous machine", which is functioned as the power controller between EVs and the power grid. At last, based on a Monte Carlo sampling, the simulation analysis of 50 EVs with the normal distribution of battery state of charge and average vehicle daily travel miles is carried out by using the proposed method. The results show that the proposed method can effectively classify the electric vehicles with different battery state of charge and different average vehicle daily travel miles. The parameters of the power converter controller for different classes of electric vehicles are optimized considering power grid frequency, their battery state of charge and their average daily travel miles, so as to maintain the balance of power grid frequency, and to meet the power needs of EV daily drive.
\end{abstract}

Keywords: electric vehicle; frequency regulation; smart grid; V2G; vehicle-to-grid; virtual synchronous machine; VSM

\section{Introduction}

With the development of electric vehicles (EVs), EVs have the potential to replace the traditional diesel locomotives and become the main means of transportation for people to travel in the future. However, the large-scale application of those battery-based electric vehicles will bring new problems to the operation and planning of existing power grids. Large-scale battery-based electric vehicle charging will result in a significant increase in grid load. If the charging behaviors occur during the peak period of the grid, it will Intensify the difference of peak-to-valley load of the current grid, hence reduces the efficiency of the current power system. For example, Reference [1] refers to a test of random charging of electric vehicles in 13 regional power grids in the United States. The results show that in order to accept high-penetrated unmanaged electric vehicles charging during early-evening, the power company needs to build an additional 160 new power stations to meet the power requirements of electric vehicles. What worse is, the peak load of traffic and power grid tends to be at the same time period, which means that there will be a large number of low-battery EVs need to be charged during the peak period of grid load. Studies used in Reference [2] show 7334 charging events of 64 EVs for a period of one year in Los Angeles, USA. It indicates that the $24 \mathrm{~h}$ charging energy distribution curve is pretty similar to the daily load curve in California. Hence, If the EV charging load is not 
well managed, once a large number of EVs that are connected to the grid at the peak time, the huge instantaneous power demand may suddenly crash the local grid. Therefore, how to control the orderly charge or discharge of a large number of EVs, so as to maintain the stability of power grids and meet the electricity needs of EV users is an important problem to be addressed and solved.

In terms of how to reduce the impact of electric vehicle charging on the grid, Reference [3] points out that in the United States, if the charging load of EVs with $10 \%$ penetration is moved from daytime to the night, it will prevent the growth of a peak load of nearly $50 \%$. Reference [4] points out that when a large number of EVs are connected to the power grid by bi-directional power electronics controller, they can be regarded as the backup storage for the power grid to deflate the energy fluctuations brought by the renewables, such as wind, solar, etc. The References $[5,6]$ pointed out that when an EV is connected to the grid by power electronic devices with bidirectional power control capability, and when the capacity of the electric vehicle is large enough, it can be regarded as the backup energy storage for the grid, to be absorbed by the renewable energy power generation system. It can absorb the excess power generated by the power generation plant, supplement the shortage of the power supply system, and thus stabilize the frequency and voltage fluctuations of the power grid. The idea of utilizing the idle power in electric vehicle batteries to feed the grid is known as vehicle-to-gird (V2G) [7].

A V2G system can provide services, such as frequency regulation, reactive power support, spinning reserve, load balancing, etc. to the power grid [8-11]. The purpose of frequency regulation to keep the grid frequency stable around the rated frequency point, so that the supply and demand balance of active power of the power system are met. As for frequency regulation, current power system mainly relies on large synchronous generators for frequency regulation by adjusting their active power output, which maintenance cost is expensive. With the increase in the number of EVs in the future, if the number is large enough, by controlling the charging and discharging behavior of the EVs group through a certain strategy, then the EVs group can be used instead of the large synchronous generators for frequency regulation. References [12-14] presents control methods to provide frequency regulation service for power grid by adjusting the charging and discharging power of EVs. Since the number of EVs in these methods [12-14] is not large, and as the number of EVs increases, the control complexity will increase exponentially, which presents a new challenge to the design of frequency regulation strategies, due to the different $\mathrm{SoC}$ and different expected time length of gird access of each electric vehicle.

To realize V2G, the bidirectional inverters, which are the interface between the electric vehicle and the power grid is the key energy exchange point and should be well controlled. At present, the inverter control method mainly considers the 'p/q method'. However, inverters controlled by $\mathrm{p} / \mathrm{q}$ method in the power grid behave like the controlled current source [15], which does not have the ability to maintain voltage and frequency stability, so p/q control does not provide effective power support in the event of a power grid failure. With the increasing penetration of distributed power sources, virtual synchronous machine (VSM) technology $[16,17]$ have received increasing attention, due to its ability to improve grid stability. VSM is a grid-friendly inverter control technology. The operating mechanism of the VSM is to simulate the inherent electromagnetic transient characteristics of the synchronous motor, so that the inverter has the same internal synchronous operation mechanism and external adjustment characteristics as the conventional synchronous generator, so that the inverter operates closer to the real synchronous generator. It can provide the necessary damping and inertia support to the grid [18], which is of great significance for future power systems with the increase of penetration rate of power electronic enabled devices in the power grid [19-21].

In this paper, VSM is utilized as the power interface between EVs and the power grid. A control method for EVs participating in grid Frequency regulation is proposed, which is based on the clustering algorithm and automatically adjust VSM parameters. Firstly, the K-means clustering algorithm is applied to cluster EVs with different battery SoC and with different average vehicle daily travel miles. Then according to the clustering results, a multi-objective optimization problem is proposed to optimize 
the set power and the droop coefficient of the VSM. The rest of this paper is organized as follows: In Section 2, K-means algorithm is introduced firstly; and then the EV group self-classify strategy is proposed; The VSM based optimal EV charging strategy is proposed in Section 3; Simulations are carried out in Section 4, and the conclusion is stated in Section 5.

\section{K-means Clustering Based EVs Classification}

\section{Introduce of K-means Clustering Algorithm}

The K-means algorithm is an iterative clustering algorithm that uses distance as a similarity indicator to discover $\mathrm{K}$ classes in a given data set, and the center of each class is based on the mean of all values in the class, each of which is described by the clustering center. For a given data set $x$ containing $\mathrm{n} \mathrm{D}$-dimensional data points and the category $\mathrm{K}$ to be divided, the Euclidean distance is selected as the similarity index, and the clustering target is to make all kinds of clustering squared and minimum, that is, to minimize:

$$
J=\sum_{k=1}^{k} \sum_{i=1}^{n}\left\|x_{i}-u_{k}\right\|^{2} .
$$

According to the least square method and Lagrange principle, the clustering center is the average of each data point in the corresponding category, and in order to make the algorithm converge, the final clustering center should be kept as constant as possible in the iterative process.

In this paper, the traditional K-means algorithm is modified to suite the control strategy proposed in Section 3, the flow of the modified K-means algorithm is as Figure 1 shown, which can be divided into six stages:

(1) Create a D-dimensional data array and import the original data;

(2) Adding K virtual standard points to a data space, Different standard points are spaced at a certain distance $\mathrm{D}$. Select the $\mathrm{K}$ virtual standard points as the initial clustering center;

(3) For other objects in the data space, calculate to their Euclidean distance by using Equation (1) from those clustering centers.

(4) According to the Euclidean distance, the objects in the D-dimensional space are divided into several classes corresponding to their closest clustering center;

(5) Update Clustering Center: The mean value of all objects in each category are defined as the new clustering center. The value of the objective function is calculated by using the clustering center of the class.

(6) Go back to step 3, calculate the distance between all objects with new clustering center, calculate new clustering center again. If the values of the clustering center have changed, then go back to step 3 again. If not, output results. 


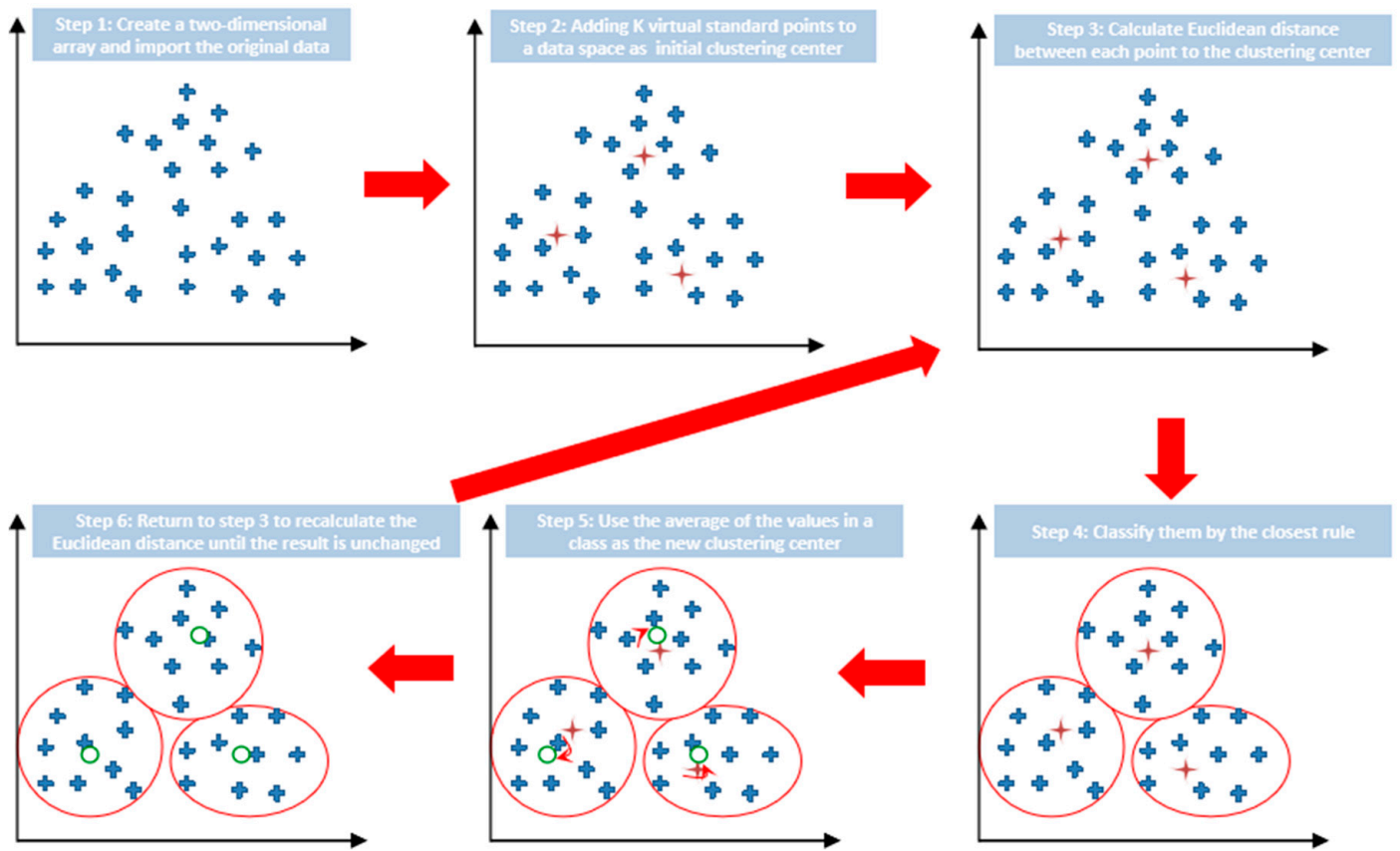

Figure 1. The calculation principle of the K-means clustering algorithm.2.2 eclectic vehicles (EVs) clustering strategy.

Because the EVs access to the power grid have a wide range of randomness and uncertainty in time and space, it is difficult to effectively manage such a huge number of random events at the same time period. Therefore, it is necessary to find a way to classify and manage these EVs according to their characteristic attributes. In this paper, a large number of EVs are classified by using the K-means classification method, the EVs are classified according to their battery SoC and average vehicle daily travel (AVDT) respectively at the first, then reorganize the EV groups by merging the EVs with the same SoC and AVDT miles into one single group. By doing so, A big electric vehicle group is divided into several sub EV groups, each sub EV group contains EVs with the same SOC and AVDT classes. The flow chart of the proposed EVs clustering strategy can be described as Figure 2 shown:

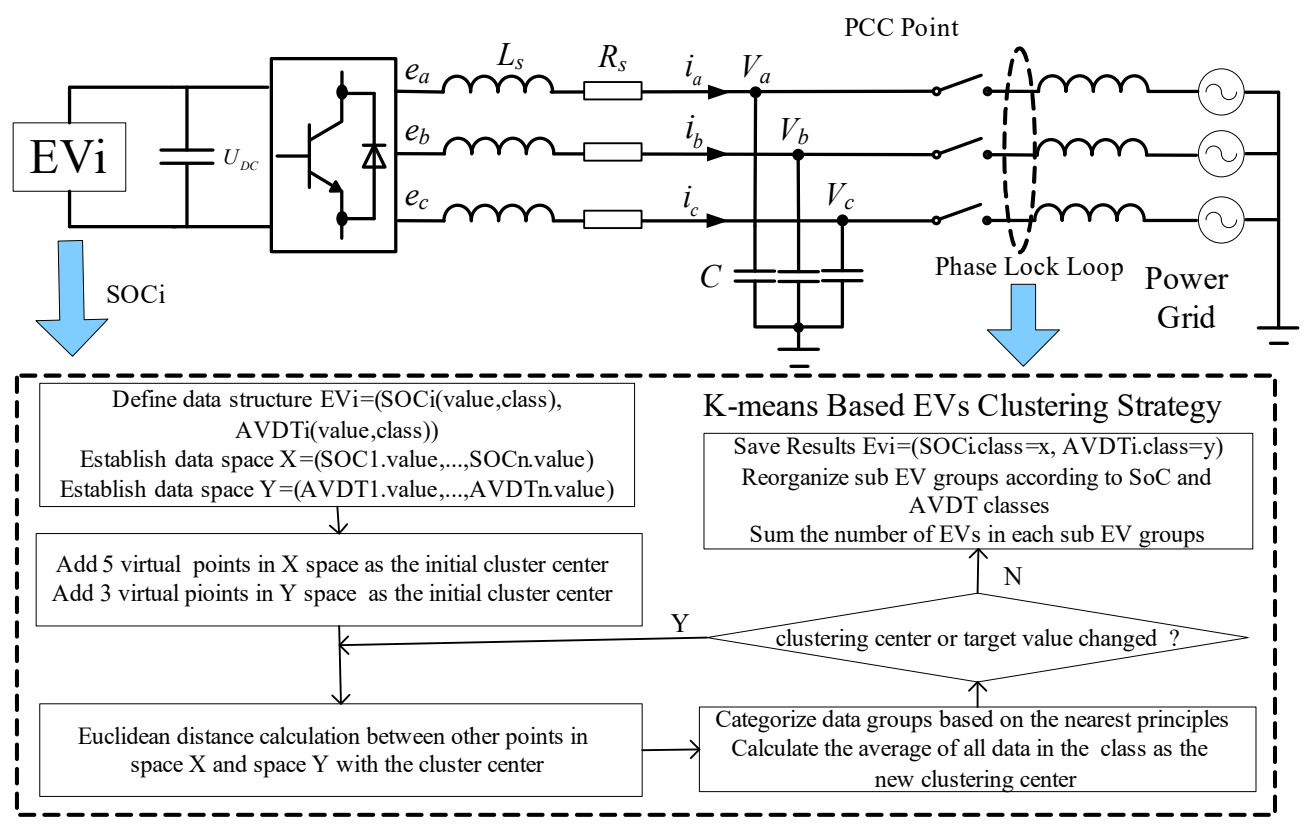

Figure 2. K-means based EVs clustering strategy. 


\section{VSM Based Optimal EV Charging Strategy}

\subsection{Brief Introduce of Virtual Synchronous Machine Technology}

Virtual synchronous machine (VSM) technology is a kind of power grid friendly inverter control method which simulates the principle design of a synchronous generator of the conventional power system [22]. The core of the virtual synchronous machine technology is to control the three-phase converter to make the converter simulate the working principle of the synchronous generator, so as to obtain the intrinsic electromagnetic conversion mechanism and external operation characteristics of the simulated synchronous generator. The virtual synchronous machine consists of two parts: The main circuit and the control system, as shown in Figure 3.

The main circuit of the virtual synchronous machine is a conventional grid-connected converter topology consisting of a DC-side power supply (which can be regarded as a prime mover), a three-phase converter, and an LC filter. The control system of the virtual synchronous machine includes the mathematical model of a virtual synchronous machine and the controller of the mathematical model.

The mathematical model of the VSM is combined with the rotor equation, torque equation, stator back EMF equation and power equation of a synchronous generator as Figure 3 shown. The controller of the mathematical model consists of a real-power to frequency droop controller and a reactive-power to voltage droop controller. The mathematical model of the VSM, as well as its' controller is embedded into the DSP controller of the three-phase converter, and the back electromotive force e is used as the PWM control signal of the three-phase converter. The output voltage of the converter is filtered by the LC filter, and the voltage on capacitor $C$ is equivalent to the virtual stator voltage.

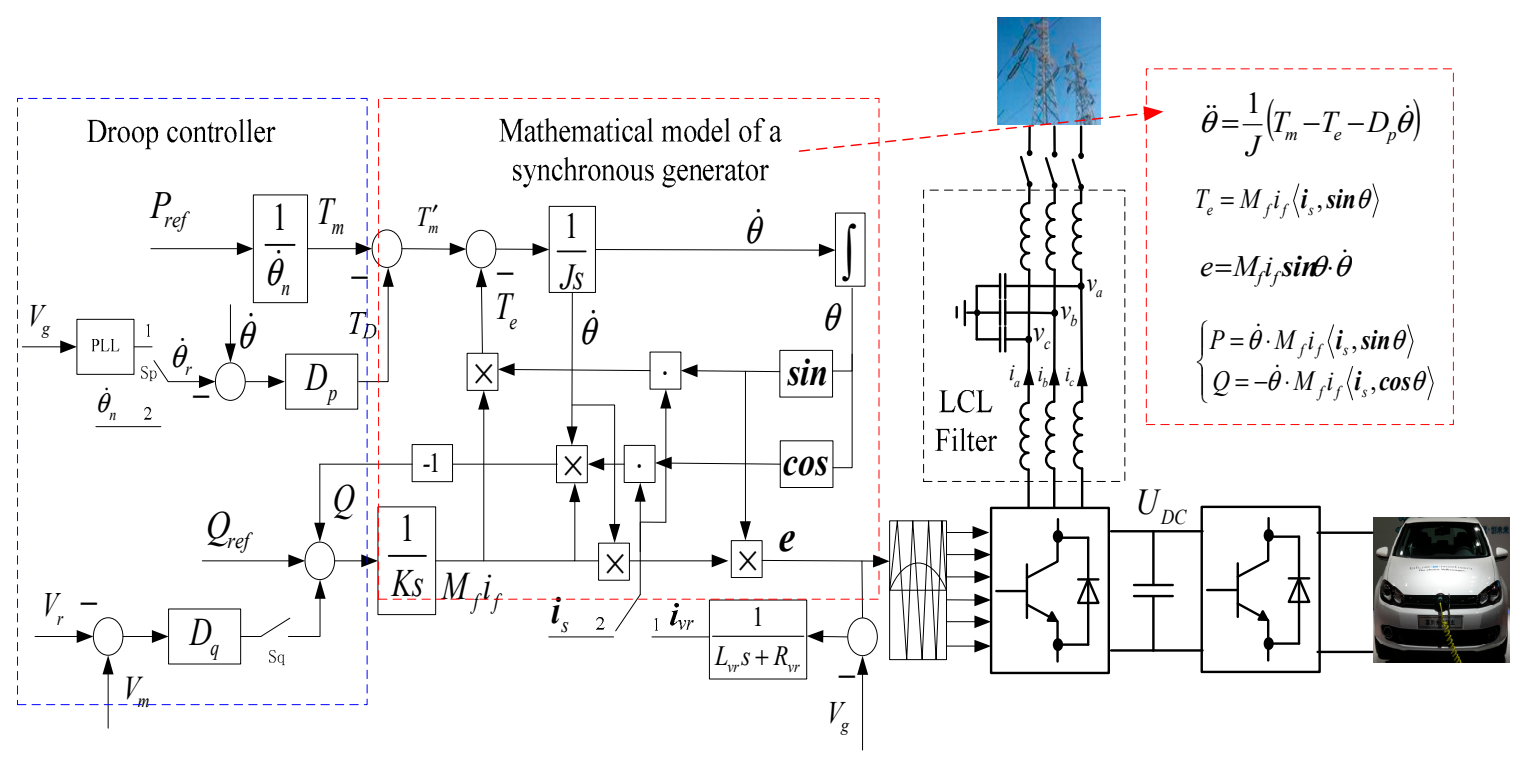

Figure 3. The topology of EV bidirectional charging circuit and its' virtual synchronous machine (VSM) controller.

\subsection{Frequency Droop Characteristic of VSM}

When the EVs are connected to the power grid through VSM, the EVs group connected to the power grid can be regarded as a synchronous generator running directly or indirectly in parallel [23], This is because the VSM simulates real synchronous generator's external characteristic. One of the outstanding advantages of this control is that the parallel bidirectional inverter of grid-connected electric vehicles can share the load proportional to their capacity, that is:

$$
m_{1} s_{1}=m_{2} s_{2}
$$




$$
n_{1} s_{1}=n_{2} s_{2}
$$

In the formula, $\mathrm{s}_{1}$ and $\mathrm{s}_{2}$ are the apparent power of two inverters, $m_{1}, m_{2}, n_{1}$ and $n_{2}$ are the droop control coefficients of the frequency control loop and the voltage control loop respectively. Set $m / n=$ C, then the voltage droop coefficient is proportional to the frequency droop coefficient, hence this paper can only focus on the frequency droop coefficient tuning:

$$
\begin{gathered}
m_{i}=-\frac{\Delta T}{\Delta \omega}=-\frac{\Delta P}{\Delta \omega \cdot \omega_{n}}=\frac{x \% \cdot S_{i}}{\left(\omega_{n}\right)^{2} \cdot y \%}=C_{p} \cdot S_{i}, \\
C_{p}=\frac{x \%}{\left(\omega_{n}\right)^{2} \cdot y \%},
\end{gathered}
$$

where $\omega_{n}$ is the rated angular frequency of power gird, if the rated power grid frequency is $50 \mathrm{~Hz}$, such as in China, then $\omega_{n}=2 \times \mathrm{pi} \times 50=314 \mathrm{rad} / \mathrm{s}$. $\mathrm{m}_{\mathrm{i}}$ means frequency fluctuations per $y \%$ result in $x \%$ of additional active power output. $S_{i}$ presents the rated capacity of a synchronous generator (SG). Apparently, in real and SG, the rated capacity of which is a definite value, but here, it is utilized as a control variable since it works in the VSM system.

As shown in Figure 4, where $P_{\text {ref }}$ is another control variable which controls the initial charging power of an EV. As $C_{p}$ can be set to a constant value, by adjusting $S_{i}$, the slope of the frequency droop characteristic of the virtual synchronous generator changes. If $P_{r e f}$ is unchanged, then the power output of EV changes linearly responding to the frequency change.

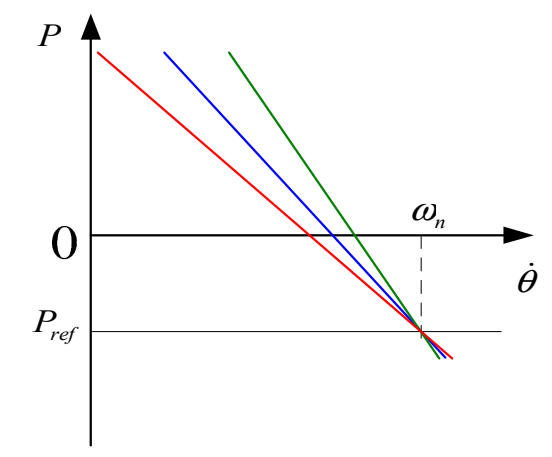

Figure 4. Frequency droop characteristic of the VSM adapts the change of $S_{i}$.

\subsection{Optimization Modeling}

According to the principal of the VSM showing in Figure 3, the charging or discharging power of $\mathrm{EV} i$ in group $j$ can be calculated as follow:

$$
P_{i, j}^{\prime}=P_{r e f . i, j}-m_{i, j} \cdot\left(\omega_{i, j}-\omega_{n}\right) \cdot \omega_{n}=P_{r e f . i, j}-C_{p} \cdot S_{i, j} \cdot \Delta \omega_{i, j} \cdot \omega_{n}
$$

where $\Delta \omega_{i, j}$ is the deviation between the local phase-locked loop detected the frequency and the rated frequency $\omega_{n} . \Delta \omega_{i, j}$ can be positive or negative, which reflect the load conditions of the power grid.

According to the clustering results introduced in Section 2, the real power of EVs group $j$ can be calculated as follows:

$$
P_{j}=\sum_{i=0}^{M} P_{i, j}^{\prime}=\sum_{i=0}^{M}\left(P_{r e f . i, j}-C_{p} S_{i, j} \Delta \omega_{i, j} \omega_{n}\right),
$$

where $M_{j}$ is the number of EVs in EV group $j$. Since the SoC and AVDT classes of EVs in group $j$ are similar, set the $M$ EVs in group $j$ has the same charging and discharging power, then Equation (7) turned to be:

$$
P_{j}=M_{j} \cdot P_{i, j}^{\prime}=M_{j} \cdot\left(P_{r e f .1 . j}-C_{p} \cdot S_{1, j} \cdot \Delta \omega_{1, j} \cdot \omega_{n}\right) .
$$


When an EV is driving on the road at a constant speed, the output power of the battery can be calculated by following the equation [24]:

$$
P_{b-o u t}=-\frac{v_{j}}{\eta_{t} \eta_{m}}\left[M g\left(f_{r}+i\right)+\frac{1}{2} \rho_{s} C_{D} A_{f} v^{2}\right] .
$$

The the negative sign in the equation is because the positive direction of power is from EVs to the grid.

In Equation (9) assuming that mechanical transmission efficiency $\eta_{t}$ is $90 \%$, motor efficiency $\eta_{m}$ is $90 \%$, vehicle weight $M=1500 \mathrm{~kg}$, road gradient $I=0$, rolling friction coefficient $f_{r}=0.012$, air density $\rho_{s}=1.29$, wind resistance coefficient $C_{D}=0.2$, windward area $A_{f}=2.0 \mathrm{~m}^{2}, v_{j}$ is the average vehicle speed of the EV, which can be calculated by [7]:

$$
v_{j}=\frac{D_{A V D T, j}}{t_{\text {ave }}}
$$

where $D_{A V D T, j}$ is the value of AVDT miles of clustering center of EV group $j . t_{\text {ave }}$ is the average time cost of the EVs which are on the road. The energy need of EV $i$ in EV group $j$ can be modeled as:

$$
E_{i, j, \text { need }}=E_{j, \text { need }}=P_{b-o u t, j} \cdot t_{\text {ave }}=-\frac{D_{A V D T, j}}{3.6 \cdot \eta_{t} \eta_{m}}\left[M g\left(f_{r}+i\right)+\frac{1}{2} \rho_{s} C_{D} A_{f}\left(\frac{D_{A V D T, j}}{3.6 \cdot t_{\text {ave }}}\right)^{2}\right]
$$

In the power system, the imbalance of active power causes the system frequency to change, which also means that the system frequency change reflects the imbalance of the system's active power. The process of large generator units adjusting the frequency of the power grid can be approximated by a linear mathematical formula as:

$$
q=\frac{\Delta P}{\Delta f}=\frac{\Delta P}{\left(f_{g}-f_{n}\right)}=\frac{\Delta P}{\Lambda \omega / 2 \pi}
$$

where $f_{g}$ is the local grid frequency of the power grid detected by the phase lock loop (PLL), $f_{n}$ is the rated grid frequency. $\Delta \mathrm{P}$ is the corresponding active power needed by the power grid to balance the system frequency in response.

Then, a multi-objective optimal problem can be formulated as follows:

$\mathrm{J} 1=$ Minimize the power imbalance

$$
\operatorname{Min} J 1=\left(\Delta P+\sum_{j=1}^{N} P_{j}^{\prime}\right)^{2}=\left(q \cdot \Delta \omega / 2 \pi+\sum_{j=1}^{N}\left[M_{j}\left(P_{r e f, j}-C_{p} S_{j} \Delta \omega_{j} \omega_{n}\right)\right]\right)^{2}
$$

where $\Delta P$ is frequency regulation demand, $\Delta P$ is positive when grid frequency is higher than its' rated value and require downward frequency regulation, $\Delta P$ is negative when grid frequency is lower than its' rated value and require upward frequency regulation. $\sum_{j=1}^{N} P_{j}^{\prime}$ is the total charging/discharging power of EVs group, it can be positive or negative, depends on optimization results.

$\mathrm{J} 2=$ Minimize the energy gap between the drive needs and the charged energy

$$
\operatorname{Min} \mathrm{J} 2=\sum_{i=1}^{M_{j}} \sum_{j=1}^{N}\left(E_{i, j, \text { need }}-P_{i, j}^{\prime}\right)^{2}=\sum_{j=1}^{N} M_{j}^{2}\left(E_{j, \text { need }}-P_{j}^{\prime} T\right)^{2},
$$

where $E_{i, j, n e e d}$ is defined by Equation (11), which means daily drive energy needed by EV $i$ in EV group $j . T$ is the average time that $\mathrm{EV}$ connected to the power grid. $P_{i, j}^{\prime} \cdot T$ means the energy $E V_{i, j}$ get from the power grid. 
Equations (13) and (14) are subject to constraints as follow:

(1) Capacity constraint

$$
S_{j, \min } \leq S_{j} \leq S_{j, \max }
$$

where $S_{j, \min }$ is the minimum capacity of VSM of EV group $j, S_{j, \max }$ is the maximum capacity of VSM of EV group $j$.

(2) Power constraint

$$
P_{i, j, \min }^{\prime} \leq P_{i, j}^{\prime}=P_{r e f}-C_{p} \cdot S_{j} \cdot \Delta \omega_{j} \cdot \omega_{n} \leq P_{i, j, \max ^{\prime}}^{\prime}
$$

where $P_{i, j, \min }^{\prime}$ is the minimum charging or discharging power of EV $i$ in group $j, P_{i, j, \text { max }}^{\prime}$ is the maximum charging or discharging power of $\mathrm{EV} i$ in group $j$.

\subsection{Multi-Objective Particle Swarm Optimization (MOPSO) Algorithm}

MOPSO Algorithm is adopted here to solve the optimization problem above [25]. The advantages of the MOPSO used in this paper are easy to implement, fast convergence (compared with NSGA-II method), and good distribution of solutions (compared with original MOPSO). The pseudo code of the MOPSO algorithm is as Algorithm 1 shows, which is mainly consist of three part:

(1) Particle location update

The system initially generates a set of particles randomly, each particle $i$ having random positional and speed information. In each iteration, if the current position of the particle is a dominated solution, the particle changes its position based on its best position in history and the global optimal position stored in the external elite file. The update rule is as line 5 to line 11 of Algorithm 1 shown.

(2) External repository file

External repository file is to preserve the non-dominated solution during the evolution process. After updating the speed and position information, each generation of particles compared with the solutions in the repository file. If the position of the particle is better than (dominant) the solution in the repository file, or the particle and the solution in the repository file do not dominant each other, then the particle is added to the repository file; if the particle is dominated by the solutions in the repository file, the elite file remains unchanged. The updating of the repository file is as line 16 to line 23 of Algorithm 1 shown.

(3) Grid crowding rejection

Grid crowd rejection mechanism is adopted for maintenance of the repository file. The purpose of grid crowd rejection is to maintain the scale of solutions in the repository file the same, and to ensure good distribution of the solutions. According to the extreme values of all non-dominated solutions in the repository file, the s-dimensional solution space in the repository file can be divided into $\mathrm{d}^{\mathrm{k}}$ grid spaces, where $d$ is the number of grids in each dimension solution space.

After the s-dimensional solution space is divided into $d^{k}$ grid spaces, Select the grid with the largest number of particles and then randomly delete one of the solutions, hence to keep the external repository size unchanged. Grid crowding rejection is as line 24 to line 27 of Algorithm 1 shown.

Processing the outputted optimal solution set by using a weighted scale method:

First, determine the weight of the two optimization targets of power imbalance and EV energy demand $\mathrm{w}_{\mathrm{r}}=\left(\mathrm{w}_{1} ; \mathrm{w}_{2}\right)$;

Then, since the units of the two optimization targets are different, they are normalized by:

$$
\bar{f}_{k, r}(x)=\frac{f_{k, r}(x)-\min _{k=1,2, \ldots, A_{\max }} f_{k, r}(x)}{\max _{k=1,2, \ldots, A_{\max }} f_{k, r}(x)-\min _{k=1,2, \ldots, A_{\max }} f_{k, r}(x)} r=1,2,
$$

where $A_{\max }$ is the number of outputted optimal solutions. 


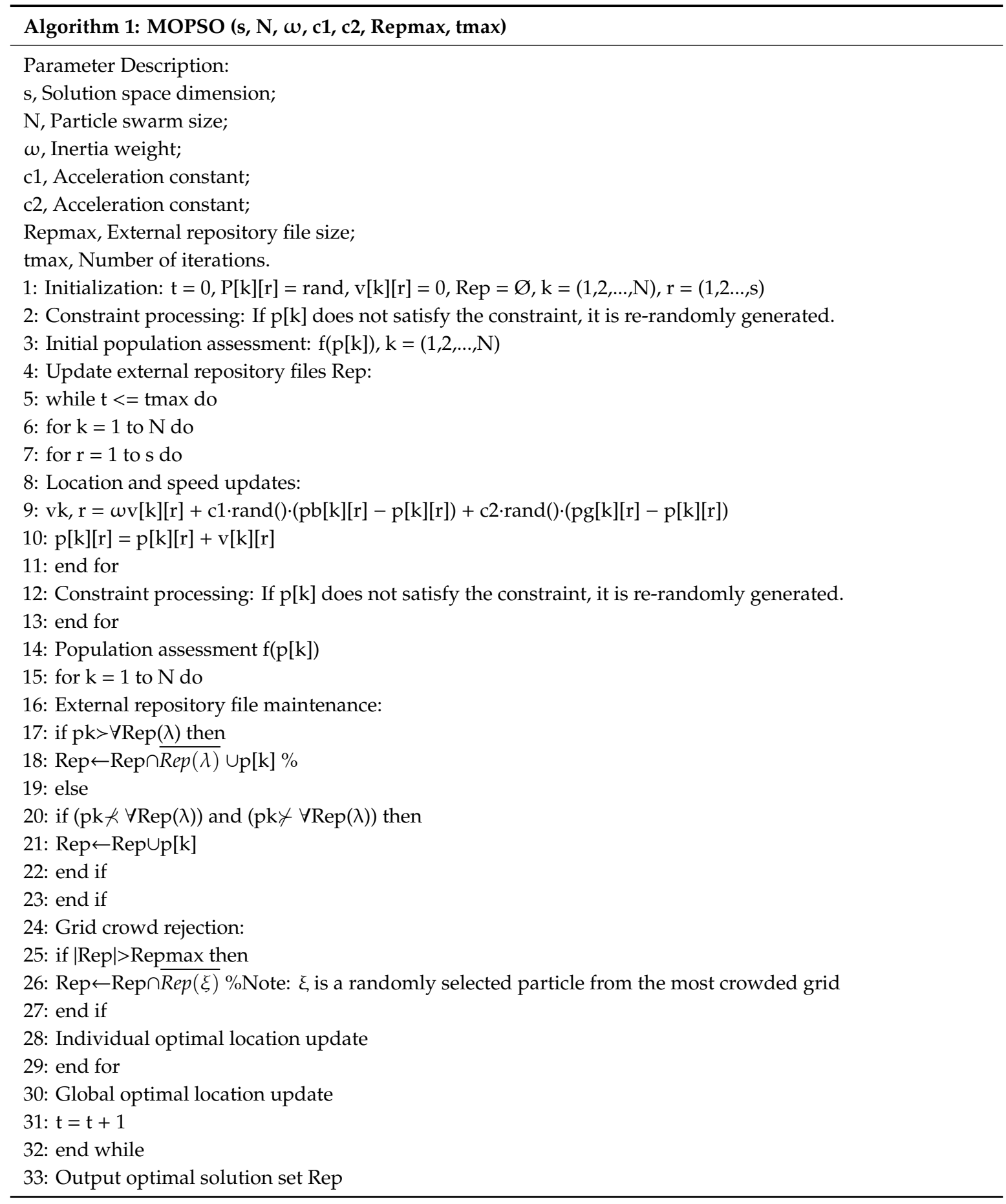

At last, calculate the weighted scale value of the normalized Pareto optimal solution by using Equation (18), sort and select the solution with the smallest weighted scale value as the compromised optimal solution:

$$
l_{k}(x)=\sqrt{\sum_{r=1}^{2} w_{r}\left|\bar{f}_{k, r}(x)-z_{r}\right|^{2}} k=1,2, \ldots, A_{\max } .
$$

In the formula, $z_{r}$ is the ideal solution of the model. Since the multi-objective optimization model established in this paper is a minimization problem, $z_{r}=0$ is taken. 


\section{Results and Discussion}

In this paper, a Matlab/Simulink simulation platform is used for simulation calculation and algorithm verification. The version of Matlab is R2017b. The CPU of the computer is Intel Core (TM) i5-8250U with $1.80 \mathrm{GHz}$ frequency. The RAM of the computer is $8.00 \mathrm{~GB}$ and the operating system is Windows 10. Using the Monte Carlo sampling method [14], the random distribution model of SoC and AVDT of 50 electric vehicles was established. The rated capacity of each EV's power battery is 100 Ah, the battery end voltage is $300 \mathrm{~V}$; The minimum spacing between different SoC classes D $1=30 \%$; The minimum spacing between different AVDT classes D2 $=20 \mathrm{~km}$; The clustering results of the 50 electric vehicles according to their SoC and AVDT classes are, as shown in Table 1 and Figure 5.

Table 1. Random models of SoC and average vehicle daily travel (AVDT) of $50 \mathrm{EVs}$ and their clustering results.

\begin{tabular}{|c|c|c|c|c|c|c|c|c|c|c|}
\hline$\overline{E V i}$ & 1 & 2 & 3 & 4 & 5 & 6 & 7 & 8 & 9 & 10 \\
\hline SoCi.value (\%) & 97 & 55 & 52 & 23 & 49 & 63 & 68 & 39 & 37 & 99 \\
\hline AVDTi.value (km) & 32 & 19 & 9 & 21 & 24 & 6 & 30 & 11 & 19 & 29 \\
\hline SoCi.class & 2 & 3 & 3 & 1 & 3 & 3 & 3 & 3 & 1 & 2 \\
\hline AVDTi.class & 1 & 3 & 2 & 3 & 3 & 2 & 1 & 2 & 3 & 1 \\
\hline EVi & 11 & 12 & 13 & 14 & 15 & 16 & 17 & 18 & 19 & 20 \\
\hline SoCi.value (\%) & 3 & 89 & 92 & 80 & 9 & 26 & 33 & 68 & 13 & 72 \\
\hline AVDTi.value (km) & 12 & 14 & 31 & 13 & 42 & 50 & 37 & 17 & 29 & 5 \\
\hline SoCi.class & 1 & 2 & 2 & 2 & 1 & 1 & 1 & 3 & 1 & 3 \\
\hline AVDTi.class & 3 & 3 & 1 & 3 & 1 & 1 & 1 & 3 & 1 & 2 \\
\hline EVi & 21 & 22 & 23 & 24 & 25 & 26 & 27 & 28 & 29 & 30 \\
\hline SoCi.value (\%) & 10 & 66 & 49 & 78 & 72 & 91 & 89 & 33 & 70 & 19 \\
\hline AVDTi.value (km) & 46 & 44 & 41 & 13 & 30 & 1 & 21 & 15 & 8 & 9 \\
\hline SoCi.class & 1 & 3 & 3 & 2 & 3 & 2 & 2 & 1 & 3 & 1 \\
\hline AVDTi.class & 1 & 1 & 1 & 3 & 1 & 2 & 3 & 3 & 2 & 2 \\
\hline EVi & 31 & 32 & 33 & 34 & 35 & 36 & 37 & 38 & 39 & 40 \\
\hline SoCi.value (\%) & 13 & 75 & 50 & 48 & 91 & 61 & 62 & 86 & 81 & 58 \\
\hline AVDTi.value (km) & 21 & 4 & 30 & 24 & 35 & 35 & 32 & 1 & 3 & 16 \\
\hline SoCi.class & 1 & 2 & 3 & 3 & 2 & 3 & 3 & 2 & 2 & 3 \\
\hline AVDTi.class & 3 & 2 & 1 & 3 & 1 & 1 & 1 & 2 & 2 & 3 \\
\hline EVi & 41 & 42 & 43 & 44 & 45 & 46 & 47 & 48 & 49 & 50 \\
\hline SoCi.value (\%) & 18 & 24 & 89 & 2 & 49 & 16 & 98 & 71 & 50 & 47 \\
\hline AVDTi.value (km) & 27 & 33 & 20 & 41 & 36 & 49 & 27 & 16 & 5 & 31 \\
\hline SoCi.class & 1 & 1 & 2 & 1 & 3 & 1 & 2 & 3 & 3 & 3 \\
\hline AVDTi.class & 1 & 1 & 3 & 1 & 1 & 1 & 1 & 3 & 2 & 3 \\
\hline
\end{tabular}

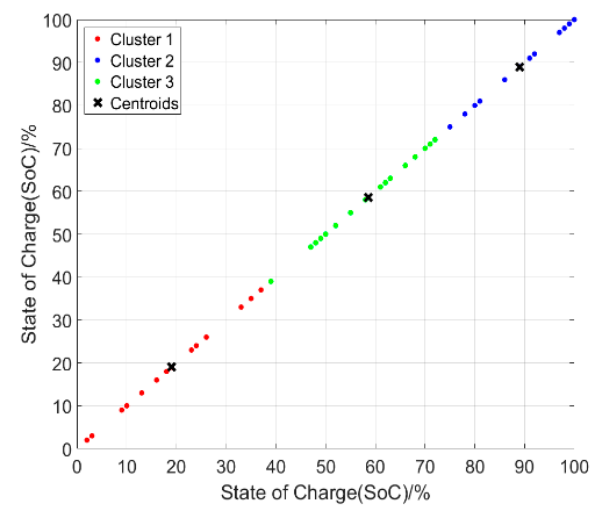

(a)

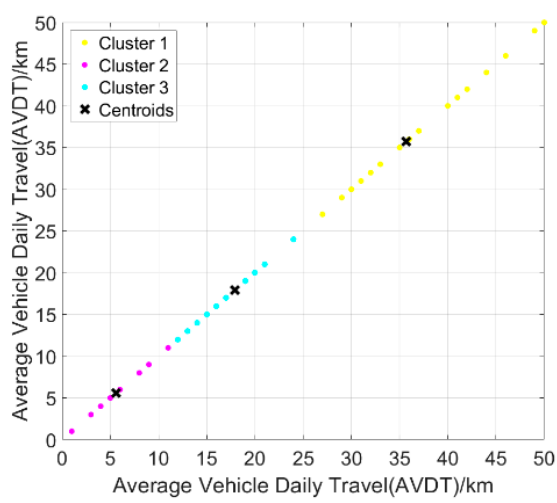

(b)

Figure 5. (a) Clustering results of EVs based on the state of charge value of each EV; (b) Clustering results of EVs based on the average vehicle daily travel value of each EV. 
Reorganize the sub EV groups according to SoC and AVDT classes, the re-organized clustering results are shown in Table 2 and Figure 6:

Table 2. Reorganized EV Groups.

\begin{tabular}{cc}
\hline Class $_{\mathbf{i}}$ & EV Groups \\
\hline SoCi.class $=$ 1, AVDTi.class $=1$ & EV15, EV16, EV17, EV19, EV21, EV41, EV42, EV44, EV46 \\
\hline SoCi.class $=$ 1, AVDTi.class $=2$ & EV30 \\
\hline SoCi.class $=$ 1, AVDTi.class $=3$ & EV4, EV9, EV11, EV28, EV31 \\
\hline SoCi.class $=$ 2, AVDTi.class $=1$ & EV1, EV10, EV13, EV35, EV47 \\
\hline SoCi.class $=$ 2, AVDTi.class $=2$ & EV26, EV32, EV38, EV39 \\
\hline SoCi.class $=$ 2, AVDTi.class $=3$ & EV12, EV14, EV24, EV27, EV43 \\
\hline SoCi.class $=3$, AVDTi.class $=1$ & EV7, EV22, EV23, EV25, EV33, EV36, EV37, EV45 \\
\hline SoCi.class $=3$, AVDTi.class $=2$ & EV3, EV6, EV8, EV20, EV29, EV49 \\
\hline SoCi.class $=3$, AVDTi.class $=3$ & EV2, EV5, EV18, EV34, EV40, EV48, EV50 \\
\hline
\end{tabular}

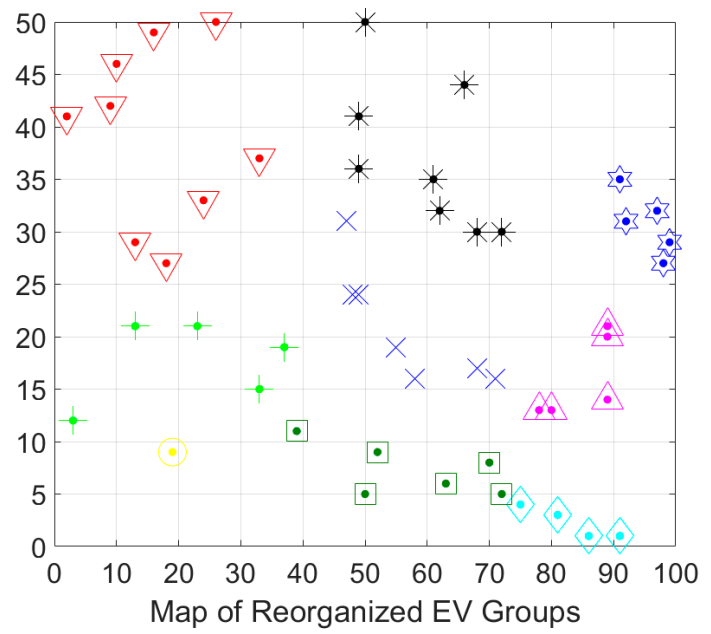

Figure 6. Auto-classified electric vehicle group map.

After completing the electric vehicle group classification, the optimization problems proposed by Equations (13) and (14) are solved, and the optimal $S_{i}$ values of the VSMs belonging to different electric vehicle groups are obtained.

In order to simplify the solution process, the " $q \cdot \Delta \omega / 2 \pi$ " term in Equation (13) is rewritten as:

$$
\Delta P_{j}=\left\{\begin{array}{cc}
q \cdot \Delta \omega_{j} \cdot \frac{M_{j} \cdot S o C_{j}}{2 \pi \cdot \sum_{j=1}^{N} M_{j} S o C_{j}} & \Delta \omega_{j} \leq 0 \\
q \cdot \Delta \omega_{j} \cdot \frac{M_{j} \cdot\left(1-S o C_{j}\right)}{2 \pi \cdot \sum_{j=1}^{N} M_{j}\left(1-S o C_{j}\right)} & \Delta \omega_{j}>0
\end{array},\right.
$$

where $M_{j}$ is the EV numbers in cluster $j$, and $S_{o} C_{j}$ means the EV SoC corresponding to each type of cluster center. $\Delta P_{j}$ should choose the upper part of Equation (19) since EVs with higher SoC have more margins for lowering charging power when $\Delta \omega_{j}$ is negative, which means the grid frequency is lower than its rated value. Relatively, $\Delta P_{j}$ should choose the lower part of Equation (19) since EVs with lower SoC have more margins for raising charging power when $\Delta \omega_{j}$ is positive.

Then, the optimization problems proposed by Equations (13) and (14) can be decomposed into 9 independent optimization problems and solved separately in this study. 
Set $x \%=100 \%, y \%=0.5 \%, C p=0.002, q=500 \mathrm{~kW} / \mathrm{Hz}, \Delta f=0.02 \mathrm{~Hz}, S_{i, \max }=5000$, $S_{i, \text { min }}=20,000, P_{\text {ref, } \max }=5000, P_{\text {ref, } \text { min }}=-5000, T=t_{\text {ave }}=1 \mathrm{~h}$, the weight of the two optimization targets $w_{r}=\left(w_{1} ; w_{2}\right)=(0.4 ; 0.6)$, the map of pareto front of the nine independent optimization problems are as Figure 7 shown.

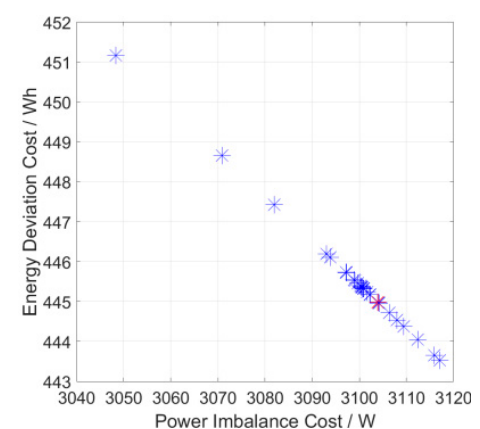

(a)

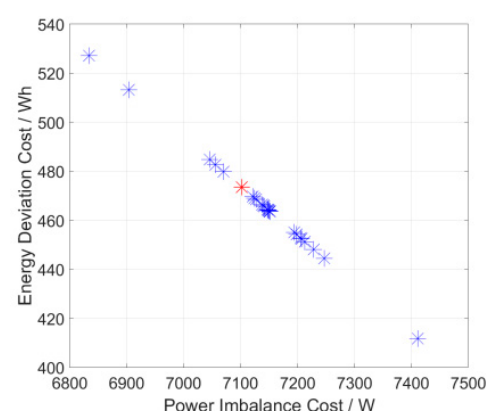

(d)

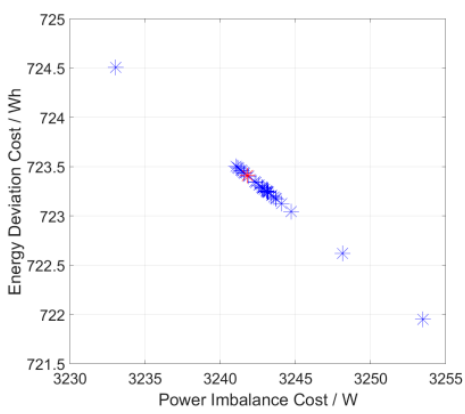

(g)

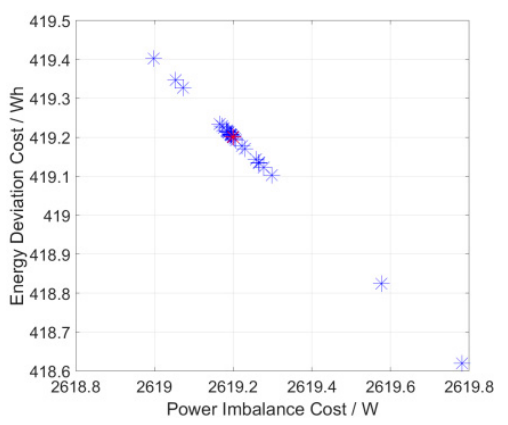

(b)

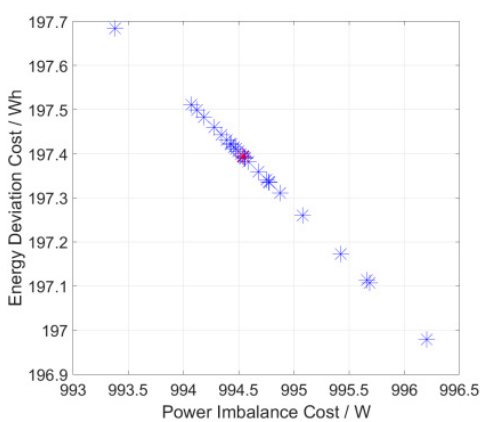

(e)

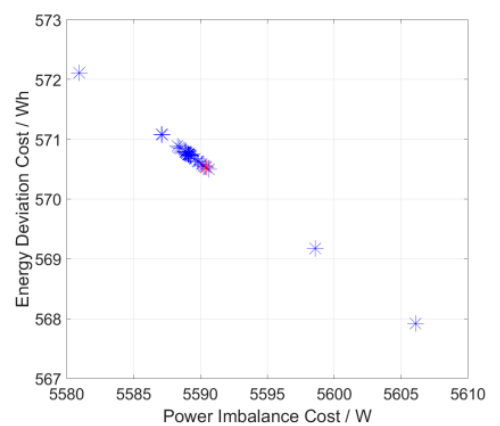

(h)

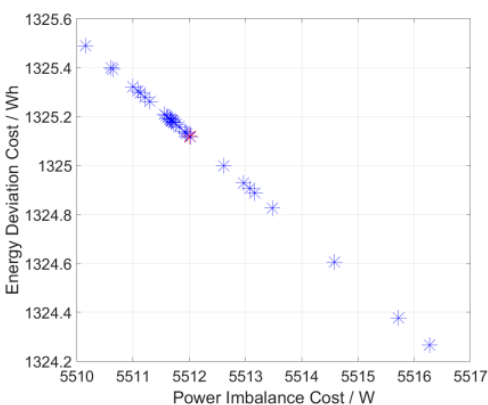

(c)

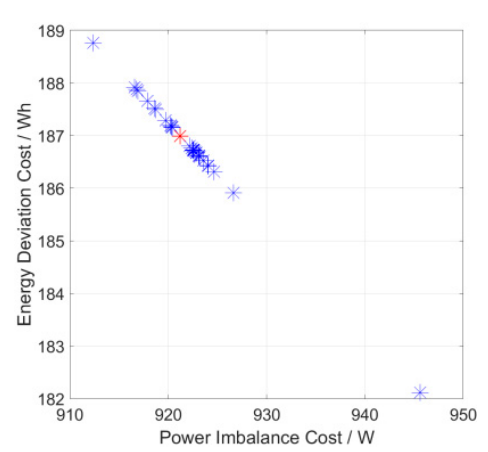

(f)

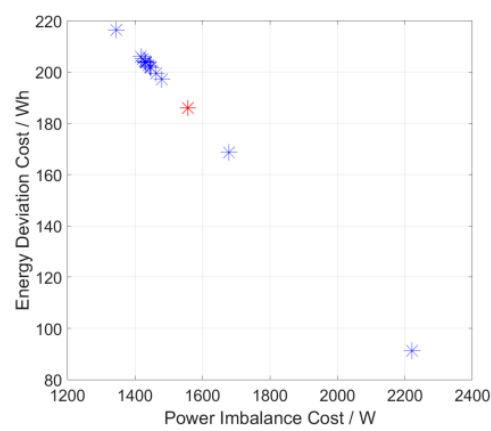

(i)

Figure 7. Pareto Front of EVs group of: (a) SoCi.class $=1$,AVDTi.class $=1$; (b) SoCi.class $=1$,AVDTi.class $=2$; (c) SoCi.class = 1,AVDTi.class = 3; (d) SoCi.class = 2,AVDTi.class = 1; (e) SoCi.class = 2,AVDTi.class = 2; (f) SoCi.class = 2,AVDTi.class = 3; (g) SoCi.class = 3,AVDTi.class = 1; (h) SoCi.class = 3,AVDTi.class = 2; (i) SoCi.class $=3$,AVDTi.class $=3$.

The optimization is indicated in Figure 7 shows the Pareto Front of the nine EVs groups. The optimization results of each electric vehicle group are independent. Each figure is the best solution for the nine EV groups (Nine maps correspond to the nine groups). By applying Equation (17) and Equation (18), the best compromised solution is selected from the pareto front, which is marked red in Figure 5. The value of the nine compromised solution, corresponding to the nine EV groups, are as Table 3 shown. Note that the total charging power is determined by both $\operatorname{Pref}_{i}$ and $S_{i}$ according to Equation (6), where $\operatorname{Pref}_{i}$ determines the initial charging power and $S_{i}$ determines the power adjustment rate according to the grid frequency deviation. From Table 3, it can be seen that, 
as for SoCi.class $=1$, which means the state of charge is relatively low, to minimize the energy gap between the drive needs and EVs charging volume, the total charging power of this class is relatively high; as for SoCi.class $=2$, which means the state of charge is relatively high, so that the total charging power of this class is relatively low. As for SoCi.class = 3, the total charging power of this class is medium. As for $\mathrm{AVDT}_{\mathrm{i}}$.class = 1, which means average daily drive distance is long, hence the total charging power of this class is relatively higher compared with EVs in the same SOC class and different AVDT class. As for $\mathrm{AVDT}_{\mathrm{i}}$.class = 2, which means average daily drive distance is short hence the total charging power of this class is relatively lower compared with EVs in the same SOC class and different AVDT class.

Test the simulation case on the Matlab/Simulink simulation platform. The circuit parameters, as shown in Figure 1 are listed in Table 4.

Table 3. Optimal Solution and Relative Costs.

\begin{tabular}{ccccc}
\hline \multicolumn{1}{c}{ Class $_{\mathbf{i}}$} & Pref $_{i}$ & $S_{i}$ & Cost1 & Cost2 \\
\hline $\mathrm{SoC}_{\mathrm{i}} \cdot$ class $=1, \mathrm{AVDT}_{\mathrm{i}} \cdot$ class $=1$ & -2346 & 11,416 & 3100 & 445 \\
\hline $\mathrm{SoC}_{\mathrm{i}} \cdot$ class $=1, \mathrm{AVDT}_{\mathrm{i}} \cdot$ class $=2$ & -190 & 12,561 & 2619 & 419 \\
\hline $\mathrm{SoC}_{\mathrm{i}} \cdot$ class $=1, \mathrm{AVDT}_{\mathrm{i}} \cdot$ class $=3$ & -1618 & 15,098 & 5512 & 1325 \\
\hline $\mathrm{SoC}_{\mathrm{i}} \cdot$ class $=2, \mathrm{AVDT}_{\mathrm{i}} \cdot$ class $=1$ & -852 & 6412 & 7908 & 474 \\
\hline $\mathrm{SoC}_{\mathrm{i}} \cdot$ class $=2, \mathrm{AVDT}_{\mathrm{i}} \cdot$ class $=2$ & 283 & 12,817 & 995 & 197 \\
\hline $\mathrm{SoC}_{\mathrm{i}} \cdot$ class $=2, \mathrm{AVDT}_{\mathrm{i}} \cdot$ class $=3$ & -106 & 19,267 & 923 & 186 \\
\hline $\mathrm{SoC}_{\mathrm{i}} \cdot$ class $=3, \mathrm{AVDT}_{\mathrm{i}} \cdot$ class $=1$ & -1393 & 5458 & 3242 & 723 \\
\hline $\mathrm{SoC}_{\mathrm{i}} \cdot$ class $=3, \mathrm{AVDT}_{\mathrm{i}} \cdot$ class $=2$ & -277 & 5762 & 5592 & 570 \\
\hline $\mathrm{SoC}_{\mathrm{i}} \cdot$ class $=3, \mathrm{AVDT}_{\mathrm{i}} \cdot$ class $=3$ & -895 & 15,119 & 1580 & 183 \\
\hline
\end{tabular}

Table 4. Parameters of EVs' bi-directional charger.

\begin{tabular}{ccccccc}
\hline$V_{n}$ & $U_{d c}$ & $V_{t}$ & $R_{g}$ & $R_{s}$ & $f_{n}$ & $C$ \\
\hline $380 \mathrm{~V}$ & $700 \mathrm{~V}$ & $300 \mathrm{~V}$ & $0.135 \Omega$ & $0.045 \Omega$ & $50 \mathrm{HZ}$ & $50 \mathrm{uF}$ \\
\hline$C_{\mathrm{dc}}$ & $x \%$ & $y \%$ & $Q$ & $L_{g}$ & $L_{s}$ & $R$ \\
\hline $10 \mathrm{mF}$ & $100 \%$ & $0.5 \%$ & $100 \mathrm{Ah}$ & $0.45 \mathrm{mH}$ & $0.15 \mathrm{mH}$ & $1000 \Omega$ \\
\hline
\end{tabular}

Set the simulation timing as follows:

(1) $0-1 \mathrm{~s}$, Initialization, set grid frequency $=50.02 \mathrm{~Hz}$, inverters are grid-connected;

(2) 1s, set active power $P_{\text {ref }}$, the real power flow to EVs are as $P_{r e f}$;

(3) 2-4s, Droop mechanism enable, the charging power of EVs changes according to different $S_{i}$;

(4) 4s, end simulation.

The simulation results are as Figure 8 shows: 


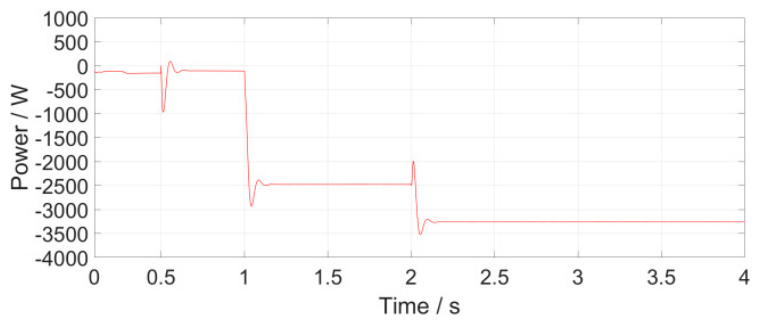

(a)

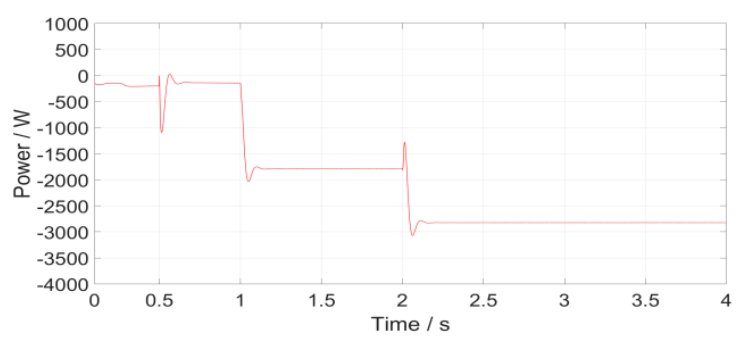

(c)

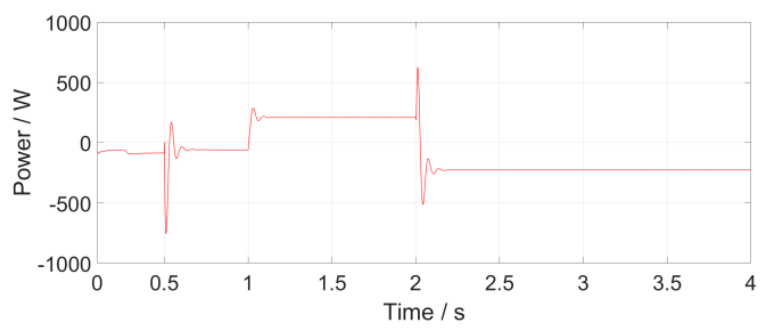

(e)

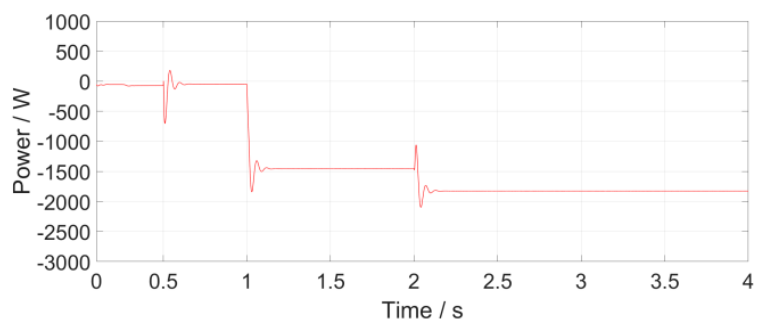

$(\mathrm{g})$

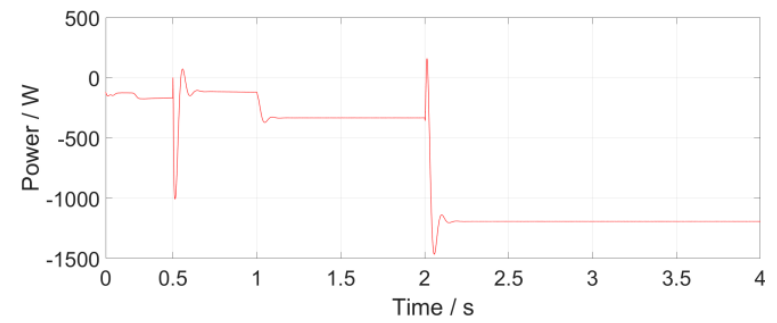

(b)

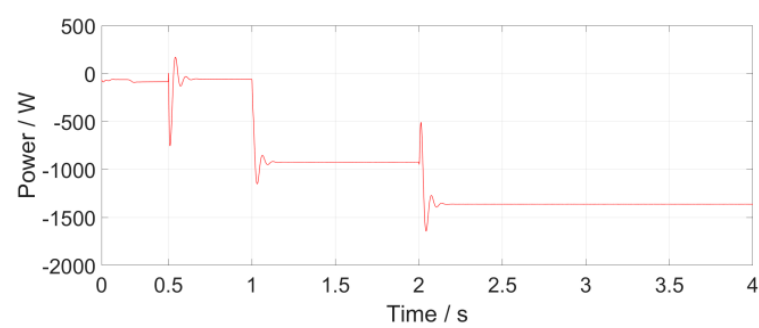

(d)

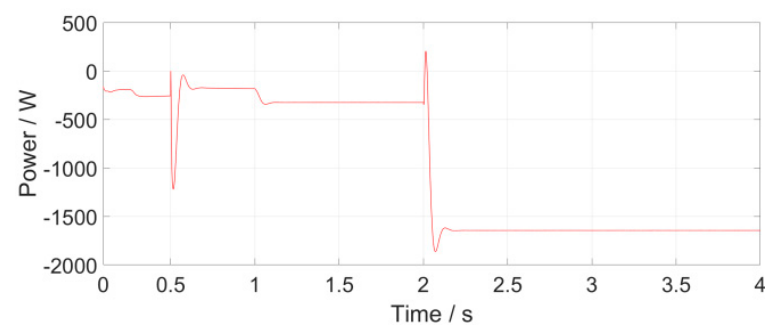

(f)

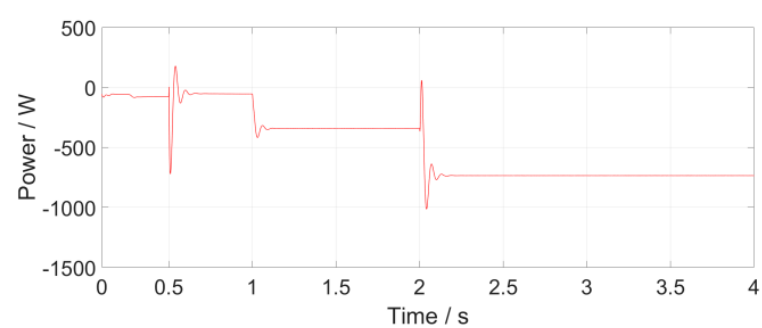

(h)

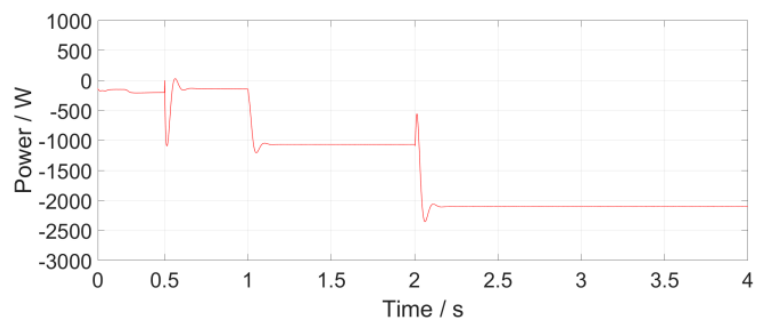

(i)

Figure 8. Charging power of $\mathrm{EV}_{\mathrm{i}, \mathrm{j}}$ in the group of: (a) SoCi.class = 1,AVDTi.class = 1; (b) SoCi.class = 1,AVDTi.class = 2; $($ c) SoCi.class $=1$,AVDTi.class $=3$; $($ d $)$ SoCi.class $=2$,AVDTi.class $=1$; (e) SoCi.class $=2$,AVDTi.class $=2 ;$ ( f SoCi.class $=2$,AVDTi.class $=3 ;$ (g) SoCi.class $=3$,AVDTi.class $=1$; (h) SoCi.class $=3$,AVDTi.class $=2$; (i) SoCi.class $=3$,AVDTi.class $=3$. 
From the simulation results, as shown in Figure 8, as the frequency of the power grid rises to $50.02 \mathrm{~Hz}$, according to the optimal results, the charging power of the EVs of the nine groups are as Pref at $t=1 \mathrm{~s}$. Then, at $t=2 \mathrm{~s}$, the droop mechanism of the virtual synchronous machine are enabled, and then it can be seen that the charging power of all groups of EVs increased again according to different $S_{i}$, which presents different droop coefficient of different virtual synchronous machines. Apparently, EVs with lower SoC have more margins for raising charging power, so when the power grid frequency is higher than its rated value, EVs with lower SoC and with higher AVDT absorb higher active power from the power grid.

If the power grid frequency is lower than its rated value, $\Delta \omega_{i, j}$, which is the deviation between the local frequency and the rated frequency, is negative. By applying the proposed optimization algorithm, Pref $_{\mathrm{i}}$ and $S_{i}$ are determined to minimize the power imbalance (J1) and the energy gap between the drive needs and the charged energy (J2) defined by Equations (13) and (14). According to Equation (13), when $\Delta \omega_{i, j}$ is negative, $\Delta \mathrm{P}$ is negative. To minimize the power imbalance, the optimization result will drive the term of $\sum_{j=1}^{N} P_{j}^{\prime}$ in Equation (13) be low. Apparently, EVs with higher SoC have more margins for lowing charging power (or discharging to the power grid), so when the power grid frequency is lower than its rated value, EVs with higher SoC and with lower AVDT absorb lower active power from the power grid (or release active power to the power grid), which would be contrary to the results of when grid frequency is high.

Establish 1000 Electric Vehicles' SoC and AVDT Models by Monte Carlo sampling Method, make the distribution of SoC and AVDT value of the EVs subject to normal distribution. The rated capacity of each EV's power battery is $100 \mathrm{Ah}$, the battery end voltage is $300 \mathrm{~V}$; The minimum spacing between different SoC classes D1 $=30 \%$; The minimum spacing between different AVDT classes D2 $=20 \mathrm{~km}$; The clustering results of the 1000 electric vehicles are as Figure 9 shown.

From Figure 9 it can be seen that, although the population of electric vehicles expands to 1000, Since the range of electric vehicle battery $\mathrm{SoC}$ is still between 0 and 100 , and the average vehicle daily travel miles is still between 0 and $50 \mathrm{~km}$, so the $1000 \mathrm{EV}$ group can still be divided into three categories respectively according to SoC and AVDT. By combining the two classifications, reorganize the sub EV groups according to SoC and AVDT classes, nine sub-EV groups are formed. Then, by applying Equation (19) to decomposition the optimization problem described by Equation (13), The solution process is disassembled to solve nine independent optimization problems. Next, only need to solve the nine independent optimization problems separately, which is no difference with the case of 50 electric vehicles as the analysis above.

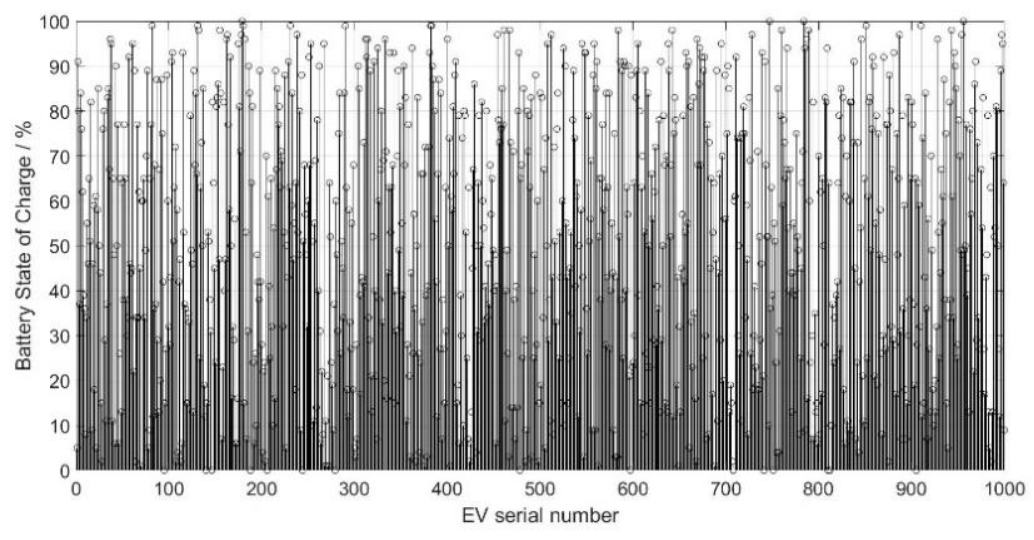

(a)

Figure 9. Cont. 


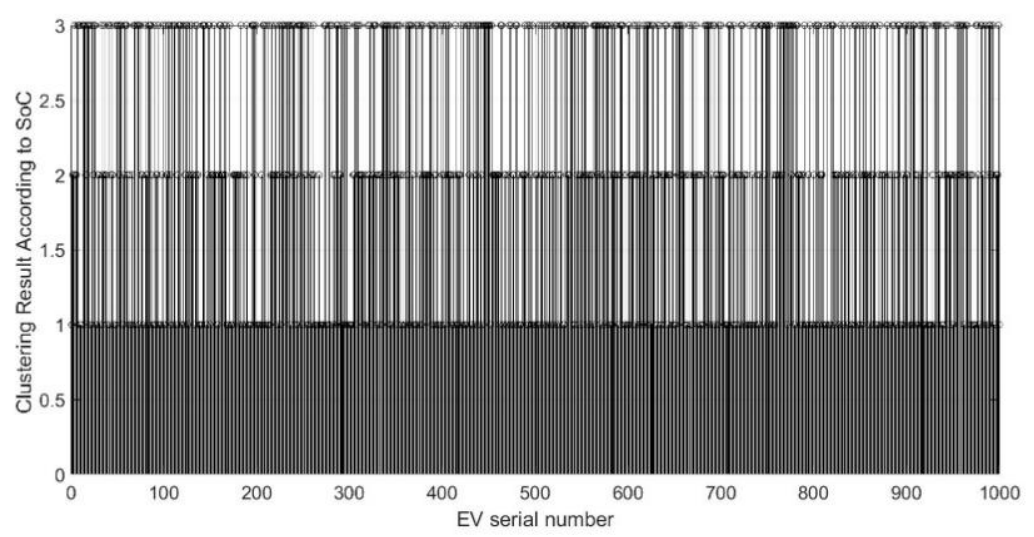

(b)

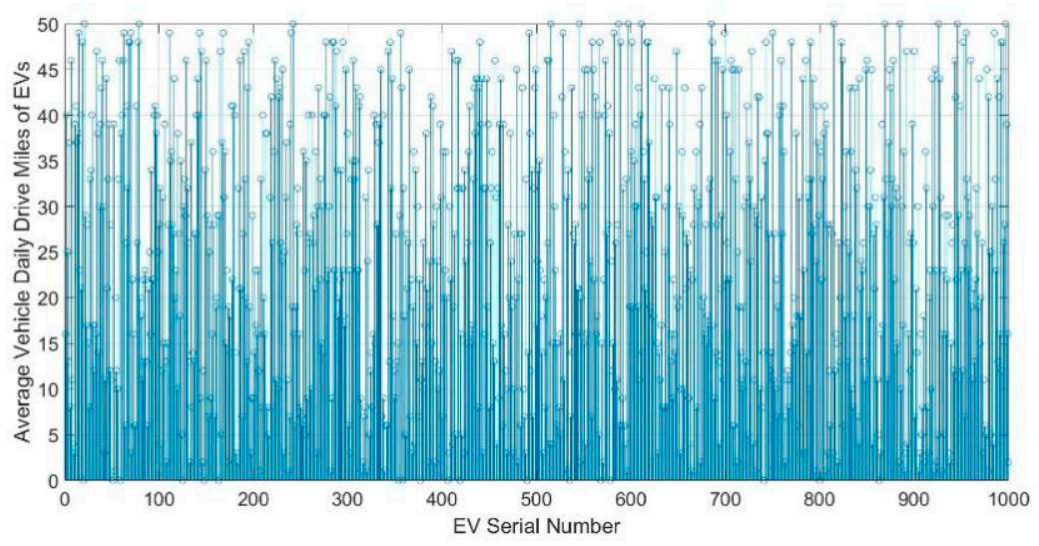

(c)

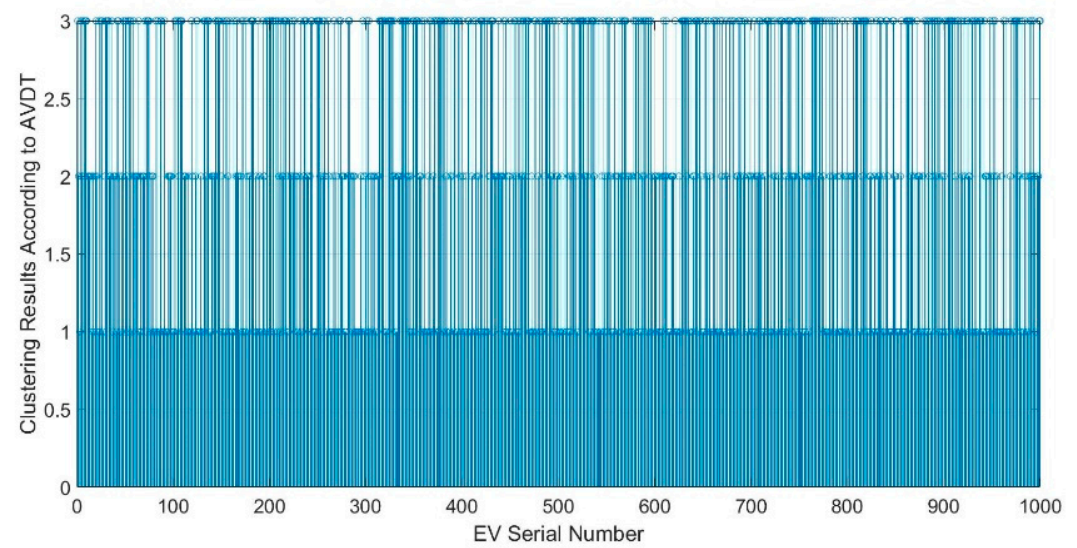

(d)

Figure 9. (a) Distribution of battery state of charge of $1000 \mathrm{EV}$; (b) Clustering results of EVs according to their SoC value; (c) Distribution of the average vehicle daily travel miles of $1000 \mathrm{EV}$; (d) Clustering results of EVs according to the average vehicle daily travel miles of $1000 \mathrm{EV}$.

\section{Conclusions}

This paper proposed a novel control method for large-scale EVs participating in grid Frequency regulation. The main achievements of this work are: (1) A classification method of electric vehicles group is proposed, which considering the battery state of charge, as well as the average vehicle daily travel miles. Because there is a certain contradiction between taking electric vehicles as energy storage to participate grid frequency regulation and as transportation tools, it is of great importance to classify electric vehicles with different battery capacity and traffic drives for the realization of the control strategy 
in this paper. (2) A multi-objective optimization model considering reducing power imbalance and feeding the driving power demand for electric vehicles. The control variable of the optimization model is the initial set power and the droop control coefficient of the "Virtual Synchronous Machine", which is like a synchronous generator with variable capacity be driven by a prime mover with adjustable output torque. This will help the power electronic converter connected to the electric vehicle to be more compatible with the synchronous generator-dominated power system. (3) The solution of optimization model based on MOPSO algorithm. For different electric vehicles group classes, the optimal compromise solution of the optimization model is calculated respectively, which are used as the control parameters to adjust the output power of the virtual synchronous machine of a different class of the electric vehicles.

Simulation results have verified the effectiveness of the proposed method. However, for the same class of electric vehicles, whether it is necessary to do a further design, so that their output power can be intelligently fine-tuned based on their more detailed power requirements, has yet to be further studied, has yet to be further studied.

Funding: This research was supported by the National Natural Science Foundation of China(Grant number 51807013). This research was supported by Hunan Provincial Natural Science Foundation of China(Grant No. 2019JJ50669). This research was Supported by Foundation of Hunan Educational Committee(Grant No. 18B137).

Conflicts of Interest: The authors declare no conflict of interest.

\section{Nomenclature}

\begin{tabular}{|c|c|}
\hline$A_{f}$ & Windward area of EV \\
\hline$C_{D}$ & Wind resistance coefficient of EV \\
\hline $\mathrm{C}_{\mathrm{p}}$ & A constant value \\
\hline $\mathrm{D}_{\mathrm{AVDT}, \mathrm{j}}$ & The value of AVDT miles of clustering center of EV group $j$ \\
\hline$E_{i, j, \text { need }}$ & The energy need of EVi in EV group $\mathrm{j}$ \\
\hline$f_{g}$ & Local detected grid frequency \\
\hline $\mathrm{f}_{\mathrm{n}}$ & The rated grid frequency \\
\hline$f_{r}$ & Rolling friction coefficient of EV \\
\hline$i$ & Road gradient of EV \\
\hline$M$ & Vehicle weight of EV \\
\hline $\mathrm{M}_{\mathrm{j}}$ & The number of EVs in EV group $\mathrm{j}$ \\
\hline $\mathrm{m}_{\mathrm{i}, \mathrm{j}}$ & The frequency droop control coefficient of EV $i$ in group $j$ \\
\hline $\mathrm{N}$ & The number of EV groups \\
\hline$P_{b-o u t}$ & Battery power consumption in EV driving \\
\hline$P_{i, j}^{\prime}$ & The charging or discharging power of EV $i$ in group $j$ \\
\hline$P_{j}$ & The charging or discharging power of EVs group $\mathrm{j}$ \\
\hline$P_{\text {ref }, i, j}$ & The initial set power of the virtual synchronous machine of EV $i$ in group $j$ \\
\hline $\mathbf{P}_{\mathrm{i}, \mathrm{j}, \min }^{\prime}$ & The minimum charging or discharging power of EV $i$ in group $j$ \\
\hline $\mathbf{P}_{i, j, \max }^{\prime}$ & The maximum charging or discharging power of EV $\mathrm{i}$ in group $\mathrm{j}$ \\
\hline$\Delta \mathrm{P}$ & Total frequency regulation demand \\
\hline$S_{i, j}$ & The capacity of the virtual synchronous machine of EV $i$ in group $j$ \\
\hline $\mathrm{S}_{\mathrm{j}, \min }$ & The minimum capacity of VSM of EV group $\mathrm{j}$ \\
\hline $\mathrm{S}_{\mathrm{j}, \max }$ & The maximum capacity of VSM of EV group $\mathrm{j}$ \\
\hline$t_{\text {ave }}$ & The average time cost of the EVs when are driving on the road \\
\hline $\mathrm{T}$ & The average time that EV connected to the power grid \\
\hline$v_{j}$ & The average vehicle speed of the EV in group $\mathrm{j}$ \\
\hline$\omega_{\mathrm{n}}$ & The rated angular frequency of the power gird \\
\hline$\Delta \omega_{i, j}$ & The frequency deviation between the value detected by EV $\mathrm{i}$ in group $\mathrm{j}$ and the rated value \\
\hline$\eta_{t}$ & Mechanical transmission efficiency of EV \\
\hline$\eta_{m}$ & Motor efficiency of EV \\
\hline$\rho_{s}$ & Air density \\
\hline
\end{tabular}




\section{References}

1. Yilmaz, M.; Krein, P.T. Review of the impact of vehicle-to-grid technologies on distribution systems and utility interfaces. IEEE Trans. Power Electron. 2013, 28, 5673-5689. [CrossRef]

2. Jiang, Z.; Tian, H.; Beshir, M.J.; Vohra, S.; Mazloomzadeh, A. Analysis of electric vehicle charging impact on the electric power grid: Based on smart grid regional demonstration project-Los Angeles. Proceeding of the 2016 IEEE PES Transmission \& Distribution Conference and Exposition-Latin America, Morelia, Mexico, 20-24 September 2016.

3. Qian, K.; Zhou, C.; Allan, M.; Yuan, Y. Modeling of load demand due to EV battery charging in distribution systems. IEEE Trans. Power Syst. 2011, 26, 802-810. [CrossRef]

4. Lund, P.D.; Lindgren, J.; Mikkola, J.; Salpakari, J. Review of energy system flexibility measures to enable high levels of variable renewable electricity. Renew. Sustain. Energy Rev. 2015, 45, 785-807. [CrossRef]

5. Peng, C.; Zou, J.; Lian, L. Dispatching strategies of electric vehicles participating in frequency regulation on power grid: A review. Renew. Sustain. Energy Rev. 2017, 68, 147-152. [CrossRef]

6. Sarabi, S.; Davigny, A.; Courtecuisse, V.; Riffonneau, Y.; Robyns, B. Potential of vehicle-to-grid ancillary services considering the uncertainties in plug-in electric vehicle availability and service/localization limitations in distribution grids. Appl. Energy 2016, 171, 523-540. [CrossRef]

7. Ahmadian, A.; Sedghi, M.; Elkamel, A.; Fowler, M.; Aliakbar Golkar, M. Plug-in electric vehicle batteries degradation modeling for smart grid studies: Review, assessment and conceptual framework. Renew. Sustain. Energy Rev. 2018, 81, 2609-2624. [CrossRef]

8. Hu, J.; Morais, H.; Sousa, T.; Lind, M. Electric vehicle fleet management in smart grids: A review of services, optimization and control aspects. Renew. Sustain. Energy Rev. 2016, 56, 1207-1226. [CrossRef]

9. Yang, Z.; Li, K.; Foley, A. Computational scheduling methods for integrating plug-in electric vehicles with power systems: A review. Renew. Sustain. Energy Rev. 2015, 51, 396-416. [CrossRef]

10. Khan, S.U.; Mehmood, K.K.; Haider, Z.M.; Bukhari, S.B.A.; Lee, S.-J.; Rafique, M.K.; Kim, C.-H. Energy management scheme for an EV smart charger V2G/G2V application with an EV power allocation technique and voltage regulation. Appl. Sci. 2018, 8, 648. [CrossRef]

11. Mao, T.; Zhang, X.; Zhou, B. Modeling and solving method for supporting 'vehicle-to-anything' EV charging mode. Appl. Sci. 2018, 8, 1048. [CrossRef]

12. Liu, H.; Hu, Z.; Song, Y.; Wang, J.; Xie, X. Decentralized vehicle-to-grid control for primary frequency regulation considering charging demands. IEEE Trans. Power Syst. 2015, 30, 3110-3119. [CrossRef]

13. Liu, D.; Zhong, Q.; Wang, Y.; Liang, Y. A synchronverter-based V2G smart charging and discharging control strategy for electric vehicles. Proc. Csee 2017, 37, 544-556.

14. Liu, D.; Zhong, Q.; Wang, Y.; Liu, G. Modeling and control of a V2G charging station based on synchronverter technology. Csee J. Power Energy Syst. 2018, 4, 326-338. [CrossRef]

15. Zhong, Q.-C.; Hornik, T. Control of Power Inverters in Renewable Energy and Smart Grid Integration; Wiley-IEEE Press: West Sussex, UK, 2013.

16. Zhong, Q.C. Power Electronics-enabled Autonomous Power Systems: Architecture and Technical Routes. IEEE Trans. Ind. Electron. 2017, 64, 5907-5918. [CrossRef]

17. Zhong, Q.C.; Konstantopoulos, G.C.; Ren, B.; Krstic, M. Improved synchronverters with bounded frequency and voltage for smart grid integration. IEEE Trans. Smart Grid 2018, 9, 786-796. [CrossRef]

18. Wu, W.; Chen, Y.; Luo, A.; Zhou, L.; Zhou, X.; Yang, L.; Dong, Y.; Guerrero, J.M. A virtual inertia control strategy for DC microgrids analogized with virtual synchronous machines. IEEE Trans. Ind. Electron. 2017, 64, 6005-6016. [CrossRef]

19. Yuan, X.; Cheng, S.; Hu, J. Multi-time scale voltage and power angle dynamics in power electronics dominated large power systems. Proc. Csee 2016, 36, 5395.

20. Freijedo, F.D.; Ferrer-Duran, M.; Dujic, D. Multi-variable high-frequency input-admittance of grid-connected converters: modeling, validation and implications on stability. IEEE Trans. Ind. Electron. 2018, 66, 6505-6515. [CrossRef]

21. Tian, X.; Wang, W.; Chi, Y.; Li, Y.; Liu, C. Virtual inertia optimisation control of DFIG and assessment of equivalent inertia time constant of power grid. IET Renew. Power Gener. 2018, 12, 1733-1740. [CrossRef]

22. Zhong, Q.C.; Nguyen, P.L.; Ma, Z.; Sheng, W. Self-Synchronized Synchronverters: Inverters Without a Dedicated Synchronization Unit. IEEE Trans. Power Electron. 2013, 29, 617-630. [CrossRef] 
23. Ying, L.; Sheng, W.; Zhong, Q.; Liu, D. Secondary Frequency Regulation Strategies and Characteristic Analysis of Synchronverter-based Microgrids. Proc. Csee 2017, 37, 391-402.

24. Ehsani, M.; Gao, Y.; Emadi, A. Modern Electric, HybridF Electric, and Fuel Cell Vehicles: Fundamentals, Theory, and Design, 2nd ed.; CRC Press: Boca Raton, FL, USA, 2005.

25. Liu, D.; Wang, Y.; Shen, Y. Electric vehicle charging and discharging coordination on distribution network using multi-objective particle swarm optimization and fuzzy decision making. Energies 2016, 9, 186. [CrossRef]

(C) 2019 by the author. Licensee MDPI, Basel, Switzerland. This article is an open access article distributed under the terms and conditions of the Creative Commons Attribution (CC BY) license (http://creativecommons.org/licenses/by/4.0/). 